\title{
Relationship between Energy Intake and Changes in Thigh Echo Intensity during the Acute Phase of Stroke in Older Patients with Hemiplegia
}

\author{
Yoji Kokura, b Mayumi Kato ${ }^{c}$ Kazuhito Kimoto ${ }^{d}$ Yoshie Okada $^{\mathrm{a}}$ Daiki Habu $^{\mathrm{b}}$ \\ aDepartment of Clinical Nutrition, Keiju Medical Center, Nanao, Japan; bepartment of Medical Nutrition, Graduate \\ School of Human Life Science, Osaka City University, Osaka, Japan; Institute of Medical, Pharmaceutical and Health \\ Sciences, National University Corporation Kanazawa University, Kanazawa, Japan; ${ }^{\mathrm{d} D e p a r t m e n t}$ of Neurology, Keiju

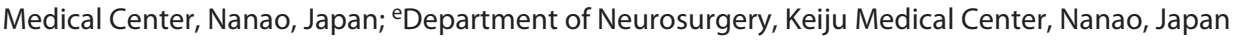

\section{Highlights of the Study}

- A decrease in skeletal muscle quality is defined as an increase in intramuscular adipose tissues, which shows as an increase in thigh echo intensity (TEI).

- The rate of change in TEI at 4 weeks after admission was $-0.9 \%$ to $+6.7 \%$.

- Higher energy intake was associated with a smaller increase in the rate of change in TEI on the nonparalyzed side.

\section{Keywords}

Adipose tissue · Cerebrovascular diseases · Quadriceps muscle · Ultrasonography

\footnotetext{
Abstract

Objective: The aim of this study was to investigate the relationship between energy intake and changes in thigh echo intensity (TEI) during the acute phase of stroke in older patients with hemiplegia. Subjects and Methods: Older hemiplegic inpatients with stroke were enrolled in this post hoc analysis of a prospective observational study. Patients were divided into 2 groups according to energy intake during the 7 days after admission as follows: energy sufficient (ES) and energy insufficient (EIS) groups. The outcome was the rate of changes in TEl of the paralyzed and nonparalyzed sides between admission and after 4 weeks. A decrease in skeletal muscle quality is defined as an increase in intramuscular adi-
}

pose tissues, which shows as an increase in echo intensity. Results: The study included 44 males and 39 females (median age 81 years). The rate of change of TEl in each group was as follows: $+4.5 \%$ in the ES/paralyzed group, $+6.7 \%$ in the EIS/ paralyzed group, $-0.9 \%$ in the ES/nonparalyzed group, and $+4.4 \%$ in the EIS/nonparalyzed group. The univariate analyses showed no significant difference in the rate of change in TEI between ES and EIS groups in both paralyzed side ( $p=$ $0.190)$ and nonparalyzed side ( $p=0.183)$. Multivariate analysis showed that higher energy intake was associated with a smaller increase in the rate of change in TEI on the nonparalyzed side ( $B=-4.115,95 \%$ confidence interval, -7.127 to -1.103). Conclusions: Higher energy intake during 7 days after admission was associated with a smaller increase in the rate of change in TEI on the nonparalyzed side upon admission and after 4 weeks.

(c) 2021 The Author(s)

Published by S. Karger AG, Basel
(C) 2021 The Author(s)

Published by S. Karger AG, Basel

This is an Open Access article licensed under the Creative Commons Attribution-NonCommercial-4.0 International License (CC BY-NC) (http://www.karger.com/Services/OpenAccessLicense), applicable to the online version of the article only. Usage and distribution for commercial purposes requires written permission.
Correspondence to:

Yoji Kokura,yojikokura@ hotmail.com 


\section{Introduction}

Recently, a decrease in skeletal muscle quality after the onset of stroke has attracted attention. Imaging tools such as magnetic resonance imaging, computed tomography, and ultrasound have been used to assess muscle quality in research settings, for example, by determining infiltration of fat into the muscle and using the attenuation of the muscle $[1,2]$. An increase in intramuscular adipose tissues is generally measured by an ultrasound scan and is expressed on the scale of echo intensity [2]. The foundational premise of echo intensity is that skeletal muscles are comprised not only of contractile proteins but also of noncontractile elements, such as intramuscular adipocytes and fibrous tissues [3]. In this study, a decrease in skeletal muscle quality is defined as an increase in intramuscular adipose tissues, which shows an increase in echo intensity.

However, the actual meaning of skeletal muscle echo intensity remains undetermined. Moreover, the foundational premise of echo intensity remains unclear [2]. The notion that fibrous tissues play a critical role in ultrasound-derived echo intensity is derived from only 2 published studies, 1 of which was conducted in animal models [4], and the other was based on autopsy findings of amyotrophic lateral sclerosis [5]. However, echo intensity measured by ultrasound has been used in some reports as an indicator of poststroke muscle quality deterioration. Increased intramuscular adipose tissues of the rectus femoris (RF) and vastus intermedius (VI) inpatients with chronic stroke are related to the reduction of muscle strength [6], decrease of gait independence [7], and risk of diabetes [1]. These observations suggest that it is important to assess thigh echo intensity (TEI) in inpatients with stroke.

The relationship between the change in TEI during the acute phase and energy intake inpatients with stroke is unclear. Few studies have investigated the changes in TEI at stroke during the acute phase; 1 previous study analyzed the long-term changes $\geq 6$ months after stroke [8]. A recent study reported that energy intake could affect the change in femur muscle thickness on the nonparalyzed limb in older inpatients with stroke [9]. Thus, the energy intake during the acute phase may be associated with muscle quality. Moreover, nutritional intake after a stroke incident is important as it promotes faster recovery and improves functional fitness [10]. However, the results of studies on nutritional intake and muscle wasting in mice are inconsistent $[11,12]$, and this relationship has not yet been investigated in humans. If the change in TEI during the acute phase is associated with energy intake, this study may extend our knowledge on the deterioration of skeletal muscle quality after stroke. Therefore, this study aimed to investigate the changes in TEI during the acute phase inpatients with stroke and to clarify the relationship between changes in TEI and energy intake.

\section{Subjects and Methods}

\section{Study Design and Subjects}

This retrospective observational study involved the post hoc analysis of data from a single center, prospective, observational, cohort study [9]. Patients with stroke who were consecutively admitted to our center, a 426-bed core hospital in a rural area were recruited between December 2016 and November 2018. The inclusion criteria were age $\geq 65$ years, an initial stroke event, and premorbid modified Rankin Scale score of 1-3. The exclusion criteria were nonparalysis of a limb, quadriplegia, medical history of intractable neurologic disease, sudden changes due to other diseases, difficulty in measuring TEI via ultrasound because of involuntary movements, death during hospitalization, and discharged during the observation period. Informed consent was obtained from all the patients or their legal guardians, and the study was approved by the institutional Ethics Committee.

Basic information was recorded for each of the included patients on admission, including age, sex, body mass index, National Institutes of Health Stroke Scale (NIHSS) score [13], comorbidity severity measured by the Charlson Comorbidity Index (CCI) score [14], swallowing ability measured using the Functional Oral Intake Scale score [15], C-reactive protein (CRP) level, nutritional status measured by the Mini Nutritional Assessment-Short Form score [16], paralyzed side, Brunnstrom stage [17], and stroke type. Further information recorded after admission included the number of days from admission to rehabilitation initiation, number of days from admission to standing initiation, rehabilitation time (in minute/day) during the 4 weeks following admission, and number of days from admission to initiation of standing.

\section{Energy and Protein Intake and Energy Sufficiency}

In this study, the energy and protein intake were calculated by averaging the daily energy and protein provided from oral, intravenous, and enteral nutrition for 7 days after admission. Energy and protein intake of patients were measured by nurses using the visual estimation method [18], and calculations were performed by a dietitian, based on the dietary intake record. Parenteral and enteral nutrition was also recorded by the dietitian. The patient's basal energy expenditure (considered to be equivalent to the resting energy expenditure) was calculated from the Harris-Benedict equation [19], which has been reported to provide the most accurate prediction of actual resting energy expenditure values for Japanese patients with acute cerebral infarction [20]. In the calculation, we used the actual body weight for patients. The patients were classified into the energy sufficient (ES) group and energy insufficient (EIS) group based on the average of the daily energy intake during the 7 days following admission. Here, we defined the "ES 
Fig. 1. The measurement areas of echo intensity of the rectus femoris and vastus intermedius. The measurement areas of echo intensity are shown within the dotted line.

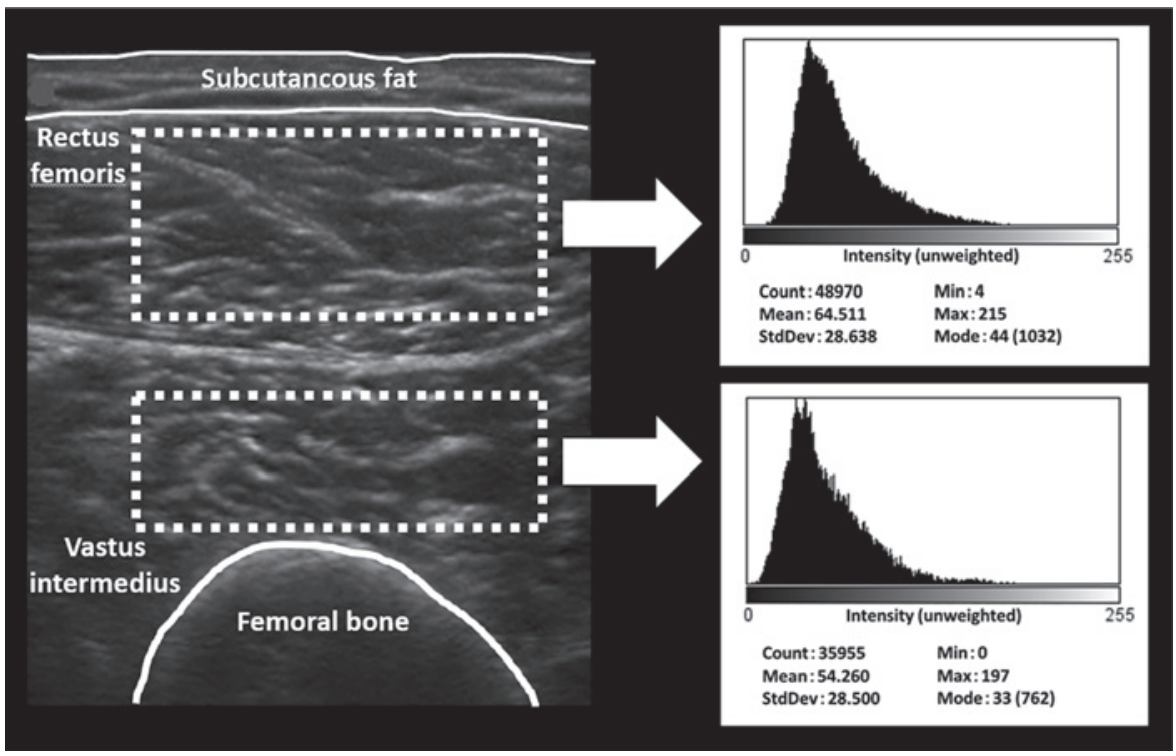

group" as those with an energy intake/basal energy expenditure of $\geq 1.0$ and the "EIS group" as those with $<1.0$.

\section{Ultrasound Measurements}

We assessed EI at the RF and VI (midway point between the anterior superior iliac spine and the proximal end of the patella [21]) in paralyzed and nonparalyzed limbs at admission and after 4 weeks using B-mode USA (SonoSite M-Turbo; Fujifilm, Tokyo, Japan) with an 8-MHz linear probe (HFL38x/13-6; Fujifilm, Tokyo, Japan). Transverse ultrasonography images of each patient were obtained with the following equipment setting: 8- $\mathrm{MHz}$ frequency, auto gain, 4.9-6 cm depth (adjusted according to the patient's muscle size [7]), and time-gain compensation in the neutral position. The measurements were obtained at rest and during maximal voluntary isometric muscle contraction with the patient in the supine position with hips and knees extended [22]. While the participants lay in a supine position with their lower limbs relaxed, a watersoluble transmission gel was applied to the skin surface of the thigh, and the probe was held perpendicular to the skin surface using the minimum pressure required to avoid deformation of the muscle and achieve a clear image [7]. All measurements were conducted by a single examiner. The intraclass correlation coefficient $(1,1)$ of TEI was 0.954 (95\% confidence interval [CI]: 0.878-0.987). All the measurements were performed 3 times. The mean values of TEI in 3 images for each RF and VI were used for further analysis. The TEI was calculated as the mean EI of the RF and VI. TEI was determined by computer-assisted 8-bit gray-scale analysis using the ImageJ software (version 1.45; National Institutes of Health, Bethesda, MD, USA). Regions of interest were determined as the specific area of the RF muscle avoiding the surrounding fascia and within the width of the femoral bone [23]. Representative ultrasonography images are shown in Figure 1. The mean TEI of the regions was expressed as a value between 0 (black) and 255 (white) [24].

\section{Outcome Measures}

The outcome was the rate of change in TEI of the paralyzed and nonparalyzed sides between admission and after 4 weeks. We cal- culated the rate of change in TEI using the following formula: rate of change in TEI $(\%)=$ (TEI after 4 weeks - TEI at admission) : $($ TEI at admission $) \times 100$.

\section{Statistical Analysis}

The statistical analyses were performed using JMP 11.2.1 software (SAS Japan, Tokyo, Japan). Continuous and ordinal data are presented as mean \pm standard deviation and median [interquartile range], and categorical data are expressed as values with percentages. The $\chi^{2}$ test, Fisher's exact test, Student's $t$ test, and the Mann-Whitney U test were used to evaluate differences between the ES and EIS groups. As this study was a post hoc analysis, the sample size could not be calculated. Therefore, we performed a post hoc power analysis using the rate of change in the TEI of the ES and EIS groups of the paralyzed and nonparalyzed sides, standard deviation, and the number of participants. Correlations were evaluated using Spearman's rank correlation analysis. Univariate analysis and multiple linear regression analysis were used to analyze whether energy sufficiency was associated with the rate of change in TEI. In this study, because the number of patients was low in relation to the number of confounders, propensity scores were used to control for confounding factors. The propensity score, as a single summary of all covariates included in the propensity score model, can be included as a covariate in a regression model of the treatment [25]. First, logistic regression analysis was performed with energy sufficiency as the objective variable and each covariate as the explanatory variables, and the propensity score was calculated. Next, multiple regression analysis was performed with propensity score and energy sufficiency as the explanatory variables and the rate of change of TEI as the objective variable. The covariates selected for multiple linear regression analysis for the rate of change in TEI were age, sex, NIHSS score, CCI score, CRP level, number of days from admission to initiation of rehabilitation, rehabilitation time during the 4 weeks following admission, and TEI at the first measurement. In all cases, $p$ values of $<0.05$ were considered statistically significant. 
Table 1. Baseline characteristics and statistical analysis with or without energy sufficiency

\begin{tabular}{|c|c|c|c|c|}
\hline Characteristic & $\begin{array}{l}\text { Total } \\
(n=83)\end{array}$ & $\begin{array}{l}\text { ES group } \\
(n=50)\end{array}$ & $\begin{array}{l}\text { EIS group } \\
(n=33)\end{array}$ & $p$ value \\
\hline Age, years & $81[75,87]$ & $81[76,86]$ & $79[69,87]$ & $0.371^{\mathrm{a}}$ \\
\hline BMI, $\mathrm{kg} / \mathrm{m}^{2}$ & $22.9 \pm 3.8$ & $22.3 \pm 3.3$ & $24.1 \pm 4.3$ & $0.033^{\mathrm{c}}$ \\
\hline Premorbid mRS & $0[0,1]$ & $0[0,1]$ & $1[0,2]$ & $0.058^{\mathrm{c}}$ \\
\hline NIHSS & $5[3,10]$ & $4[2,6]$ & $8[5,16]$ & $<0.001^{\mathrm{c}}$ \\
\hline $\mathrm{CRP}, \mathrm{mg} / \mathrm{dL}$ & $0.16[0.06,0.38]$ & $0.12[0.05,0.32]$ & $0.17[0.09,0.59]$ & $0.034^{\mathrm{c}}$ \\
\hline MNA-SF & $8[6,10]$ & $9[6,10]$ & $7[6,9]$ & $0.019^{c}$ \\
\hline \multicolumn{5}{|l|}{ Paralysis, $n(\%)$} \\
\hline Right hemiplegia & $37(45)$ & $23(46)$ & $14(42)$ & \multirow[t]{2}{*}{$0.748^{\mathrm{b}}$} \\
\hline Left hemiplegia & $46(55)$ & $27(54)$ & $19(58)$ & \\
\hline Number of days from admission to initiation of rehabilitation & $1[1,2]$ & $1[1,2]$ & $1[1,2]$ & $0.979^{c}$ \\
\hline Number of days from admission to initiation of standing & $2[1,5]$ & $2[1,4]$ & $3[2,11]$ & $0.019^{c}$ \\
\hline Rehabilitation time during the 4 weeks following admission ( $\mathrm{min} /$ day) & $119[95,139]$ & $119[94,140]$ & $120[93,140]$ & $0.907^{\mathrm{c}}$ \\
\hline
\end{tabular}

Data are expressed as mean \pm standard deviation, median [interquartile range], or number (\%). EI, energy sufficient; EIS, energy insufficient; mRS, modified Rankin Scale; NIHSS, National Institutes of Health Stroke Scale; CCI, Charlson Comorbidity Index; FOIS, Functional Oral Intake Scale; MNA-SF, Mini Nutritional Assessment-Short Form; BMI, body mass index; CRP, C-reactive protein. ${ }^{\mathrm{a}}$ Mann-Whitney U test. ${ }^{\mathrm{b}} \chi^{2}$ test. ${ }^{\mathrm{c}}$ Student's $t$ test. ${ }^{\mathrm{d}}$ Fisher's exact test.

Table 2. TEI, energy intake, and protein intake

\begin{tabular}{|c|c|c|c|}
\hline Characteristic & ES group $(n=50)$ & EIS group $(n=33)$ & $p$ value \\
\hline \multicolumn{4}{|l|}{ TEI at first measurement } \\
\hline Paralyzed side & $59.4 \pm 12.5$ & $57.9 \pm 15.0$ & $0.627^{\mathrm{a}}$ \\
\hline Nonparalyzed side & $58.4 \pm 12.1$ & $56.8 \pm 11.4$ & $0.553^{\mathrm{a}}$ \\
\hline \multicolumn{4}{|l|}{ TEI at after 4 weeks } \\
\hline Paralyzed side & $61.2 \pm 11.9$ & $62.7 \pm 15.2$ & $0.614^{\mathrm{a}}$ \\
\hline Nonparalyzed side & $58.3 \pm 11.4$ & $59.2 \pm 13.1$ & $0.754^{\mathrm{a}}$ \\
\hline \multicolumn{4}{|l|}{ Rate of change in TEI, \% } \\
\hline Paralyzed side & $+4.5[-6.3,11.2]$ & $+6.7[-1.1,16.3]$ & $0.190^{\mathrm{b}}$ \\
\hline Nonparalyzed side & $-0.9[-6.7,8.1]$ & $+4.4[-6.1,13.3]$ & $0.183^{\mathrm{b}}$ \\
\hline \multicolumn{4}{|c|}{ Energy intake (kcal/ABW/day) } \\
\hline Oral intake & $24.0[20.5-28.8]$ & $7.9[1.2-13.7]$ & $<0.001^{\mathrm{b}}$ \\
\hline Parenteral nutrition & $0[0-1.4]$ & $2.5[0.3-5.6]$ & $<0.001^{\mathrm{b}}$ \\
\hline Enteral nutrition & $0[0-0]$ & $0[0-0.6]$ & $<0.001^{\mathrm{b}}$ \\
\hline Total & $27.1 \pm 0.8$ & $13.3 \pm 1.6$ & $<0.001^{\mathrm{a}}$ \\
\hline \multicolumn{4}{|c|}{ Protein intake (kcal/ABW/day) } \\
\hline Oral intake & $1.0[0.9-1.2]$ & $0.4[0.1-0.6]$ & $<0.001^{\mathrm{b}}$ \\
\hline Parenteral nutrition & $0[0-0]$ & $0[0-0.4]$ & $<0.001^{\mathrm{b}}$ \\
\hline Enteral nutrition & $0[0-0]$ & $0[0-0]$ & $0.002^{\mathrm{b}}$ \\
\hline Total & $1.1 \pm 0.1$ & $0.6 \pm 0.1$ & $<0.001^{\mathrm{a}}$ \\
\hline
\end{tabular}

Data are expressed as mean \pm standard deviation or median [interquartile range]. ES, energy sufficient; EIS, energy insufficient; TEI, thigh echo intensity; ABW, actual body weight. ${ }^{a}$ Student's $t$ test. ${ }^{\mathrm{b}}$ Mann-Whitney U test. 
Table 3. Spearman's rank correlation coefficients between factors

\begin{tabular}{|c|c|c|c|c|c|c|c|}
\hline Factor & $\begin{array}{l}\text { NIHSS } \\
\text { score }\end{array}$ & CCI score & CRP & $\begin{array}{l}\text { Number of days } \\
\text { from admission to } \\
\text { rehabilitation } \\
\text { initiation }\end{array}$ & $\begin{array}{l}\text { Rehabilitation time } \\
\text { during the } 4 \text { weeks } \\
\text { following admission }\end{array}$ & $\begin{array}{l}\text { TEI at } \\
\text { the first } \\
\text { measurement }\end{array}$ & $\begin{array}{l}\text { Rate of } \\
\text { change in } \\
\text { TEI }\end{array}$ \\
\hline Age, years & 0.024 & 0.014 & 0.049 & -0.012 & -0.107 & $0.387^{* *}$ & 0.070 \\
\hline NIHSS score & & -0.188 & 0.145 & -0.059 & -0.038 & -0.181 & 0.078 \\
\hline CCI score & & & 0.021 & 0.162 & $-0.270^{*}$ & 0.203 & -0.066 \\
\hline Rehabilitation time during the 4 weeks following admission & & & & & & -0.183 & 0.015 \\
\hline TEI at the first measurement & & & & & & & $-0.414^{* *}$ \\
\hline \multicolumn{8}{|l|}{ Nonparalyzed side } \\
\hline Age, years & 0.024 & 0.014 & 0.049 & -0.012 & -0.107 & $0.488^{* *}$ & 0.153 \\
\hline NIHSS score & & -0.188 & 0.145 & -0.059 & -0.038 & -0.147 & -0.020 \\
\hline CCI score & & & 0.021 & 0.162 & $-0.270^{*}$ & $0.226^{*}$ & -0.032 \\
\hline
\end{tabular}

NIHSS, National Institutes of Health Stroke Scale; CCI, Charlson Comorbidity Index; CRP, C-reactive protein; TEI; thigh echo intensity. ${ }^{*} p<0.05$. ** $p<0.001$.

\section{Results}

A total of 152 patients met the inclusion criteria. Of these, 69 were excluded from the analysis; 6 did not have paralysis of a limb, 9 had quadriplegia, 1 had medical history of intractable neurological disease, 2 experienced sudden changes due to other diseases, measuring the TEI was difficult for 4 because of involuntary movements, 9 died during hospitalization, and 38 were discharged during the observation period. Hence, the final cohort included 83 patients.

The median length of time from stroke onset to hospital admission was 0 [0-0] days. The median time from hospitalization to the first measurement of TEI was 20 [9-40] h. Of the 83 patients included in the analysis, 50 (60.2\%) were included in the ES group and $33(39.8 \%)$ in the EIS group based on their intake during the 7 days after admission. Table 1 compares the characteristics of the 2 groups. The ES group had a significantly lower BMI, NIHSS score, CRP level, and number of days from admission to initiation of standing, significantly higher Functional Oral Intake Scale score, Mini Nutritional Assessment-Short Form score, and Brunnstrom stage than those of the EIS group (all $p<0.05$ ).

Table 2 shows the differences between the groups in TEI at the first measurement and at discharge, the rate of change in TEI, and energy and protein intake. In both paralyzed and nonparalyzed limbs, the rate of change in echo strength of the quadriceps was higher in the EIS group than that in the ES group, although this rate was not significant (paralyzed side: +4.5 vs. $+6.7 \%, p=0.190$, power $=0.334$; nonparalyzed side: -0.9 vs. $+4.4 \%, p=$ 0.183 , power $=0.287$ ). The energy and protein intake during the 7 days after admission was significantly higher in the ES group than in the EIS group (energy; 27.1 vs. 13.3 $\mathrm{kcal} / \mathrm{actual}$ body weight/day, $p<0.001$; protein; 1.1 versus $0.6 \mathrm{~g} /$ actual body weight/day, $p<0.001$ ).

Associations between age, NIHSS score, CCI score, $\mathrm{CRP}$, number of days from admission to initiation of rehabilitation, rehabilitation time during the 4 weeks following admission, TEI at the first measurement, and the rate of change in TEI are reported in Table 3. The rate of change in TEI was significantly correlated with TEI at the first measurement in both paralyzed and nonparalyzed limb.

After adjusting for the propensity score for covariates, the multivariate analysis of the rate of change in TEI showed that energy sufficiency was independently associated with the rate of change in TEI in the nonparalyzed limb (adjusted difference of the rate of change in TEI were $+0.6 \%$ in ES group vs. $+4.5 \%$ in EIS group; partial regression coefficient $=-4.115 ; 95 \% \mathrm{CI},-7.127$ to $-1.103 ; p=$ 0.008 ) but not in the paralyzed limb (adjusted difference of the rate of change in TEI were $+4.3 \%$ in ES group vs. 
$+9.6 \%$ in EIS group i partial regression coefficient $=$ $-3.004 ; 95 \%$ CI, -6.986 to $0.977 ; p=0.137)$. The mean values of the calculated propensity scores for the paralyzed and nonparalyzed limbs were $0.620 \pm 0.22$ and 0.624 \pm 0.22 , respectively.

\section{Discussion}

In this retrospective observational study, we investigated the changes in TEI during the acute phase inpatients with stroke and clarified their relationship with energy intake. This resulted in 2 important clinical findings. First, the rate of change in TEI was $+4.5 \%$ to $+6.7 \%$ in the paralyzed side and $-0.9 \%$ to $+4.4 \%$ in the nonparalyzed side. Second, higher energy intake during 7 days after admission was associated with smaller increase in the rate of change in TEI on the nonparalyzed side.

The rate of change in TEI was $+4.5 \%$ to $+6.7 \%$ in the paralyzed group and $-0.9 \%$ to $+4.4 \%$ in the nonparalyzed group. The lower TEI at the first measurement was associated with an increase in TEI during the follow-up. A previous study has reported a decrease of $0.8 \%$ in TEI in community-dwelling older people during a 4-year follow-up period [26]. In addition, in chronic stroke survivors, an increase of $20.0 \%$ in TEI on the paretic side and $24.9 \%$ in TEI on the nonparetic side at follow-up after 3 years was observed when compared with the baseline values [8]. The rate of change in TEI except for the ES/nonparalyzed group observed in the present study was higher than that in community-dwelling older people and lower than that in chronic stroke survivors. These results suggest that the TEI inpatients with stroke began to increase from the acute phase and that deterioration of muscle quality may accelerate toward the chronic phase. It is important for medical staff to consider changes in muscle quality inpatients with acute stroke.

According to multivariate analysis, higher energy intake during 7 days after admission was associated with a smaller increase in the rate of change in TEI on the nonparalyzed side. In a recent study, it was observed that energy intake might affect changes in femoral muscle thickness in the nonparalyzed limb in older in-patients with stroke [9]. In addition, patients who had less energy and protein intake early during their stay in an intensive care unit showed significantly greater declines in psoas muscle density (i.e., extensive fatty infiltration) [27]. Thus, higher energy intake during the acute phase of stroke may be related not only with muscle mass but also with decline in muscle quality on the nonparalyzed side. Further, higher protein intake may be associated with a smaller increase in the rate of change in TEI. Protein is important for the maintenance and growth of skeletal muscle. For older people who are malnourished or at risk of malnutrition because of an acute or chronic illness, their diet should provide $1.2-1.5 \mathrm{~g}$ protein/kg body weight/day, which is necessary to maintain muscle strength and function [28]. In the present study, the ES group with high protein intake did not show an increase in TEI on the nonparalyzed side. Therefore, higher protein intake during the acute phase of stroke may be related to a smaller increase in the rate of change in TEI on the nonparalyzed side inpatients with stroke-related hemiplegia.

On the contrary, higher energy intake was not associated with a smaller increase in the rate of change in TEI on the paralyzed side. Muscle wasting inpatients with acute and subacute stroke may result from direct metabolic, inflammatory, and hormonal signals [29]. Underfeeding leads to a reduction of insulin-like growth factor-1, which, in turn, may act via the IRS-1-PI3K/Akt signaling account for an activation of caspase-3. In stroke mice, a significant correlation was observed between the amount of food intake and insulin-like growth factor-1 levels in the control group [30]. However, this correlation was not found in sham and stroke mice, and insulin-like growth factor-1 levels were not lower. High energy feeding failed to prevent weight loss or muscle tissue wasting and increased proteolytic activity [30]. As in addition to the systemic catabolic effects, a direct and local proteolytic signal may occur after stroke, and nutritional intake cannot fully explain this catabolic activation on the paralyzed side.

We acknowledge that this study has several limitations. First, this observational study was conducted at a single hospital, which may limit the generalization of the results. Second, as this study was the post hoc analysis, the sample size could not be calculated. The univariate analysis showed no significant difference in the change in TEI between the ES and EIS groups, whereas the multivariate analysis indicated a significant difference. This result might be affected by confounders, but it may be also affected by the low statistical power in this study. Third, TEI was measured twice; the lower TEI at the first measurement was associated with an increase in TEI during the follow-up. However, lower values at admission are associated with a greater increase in TEI during the follow-up. Therefore, the average values may be influenced by regression to the mean. Finally, TEI may be affected by many factors such as age, sex, muscle size, voluntary strength, muscle damage, hydration, glycogen, device set- 
ting, and subcutaneous fat correction [2]. However, we were not able to unravel the influences of these factors on TEI in this study.

\section{Conclusion}

This study was the first to investigate the changes in TEI during the acute phase inpatients with stroke and to clarify their relationship with energy intake. Higher energy intake during 7 days after admission was associated with a smaller increase in the rate of change in TEI on the nonparalyzed side; conversely, the results suggested that energy intake was not associated with the rate of change in TEI on the paralyzed side. There is a need for further longitudinal studies to elucidate the causal relationship between decrease in muscle quality and energy intake.

\section{Statement of Ethics}

Our study was in accordance with the ethical standards of the Helsinki Declaration of 1975, as revised in 1983. This study was approved by the Institutional Review Board for Keiju Medical Center (No. 2017-3-1), and informed consent was obtained from all patients.

\section{Conflict of Interest Statement}

The authors have no conflicts of interest to disclose.

\section{Author Contributions}

Y.K. was responsible for study conception and design, data collection and interpretation, and drafting and revision of the manuscript. M.K. was responsible for study conception and design, data collection, and revision of the manuscript. K.K., and Y.O. were responsible for study conception, data collection and interpretation, and revision of the manuscript. D.H. was responsible for study conception and design, data interpretation, and revision of the manuscript. All the authors approved of the final version of the manuscript.

\section{Data Availability Statement}

The data that support the findings of this study are not publicly available due to their containing information that could compromise the privacy of research participants but are available from the corresponding author Y.K. upon reasonable request.

\section{References}

1 Ryan AS, Buscemi A, Forrester L, HaferMacko CE, Ivey FM. Atrophy and intramuscular fat in specific muscles of the thigh: associated weakness and hyperinsulinemia in stroke survivors. Neurorehabil Neural Repair. 2011 NovDec;25(9):865-72.

2 Stock MS, Thompson BJ. Echo intensity as an indicator of skeletal muscle quality: applications, methodology, and future directions. Eur J Appl Physiol. 2021 Feb;121(2):369-80.

3 McGregor RA, Cameron-Smith D, Poppitt $\mathrm{SD}$. It is not just muscle mass: a review of muscle quality, composition and metabolism during ageing as determinants of muscle function and mobility in later life. Longev Healthspan. 2014 Dec 1;3(1):9.

4 Pillen S, Tak RO, Zwarts MJ, Lammens MM, Verrijp KN, Arts IM, et al. Skeletal muscle ultrasound: correlation between fibrous tissue and echo intensity. Ultrasound Med Biol. 2009 Mar;35(3):443-6.

5 Arts IM, Schelhaas HJ, Verrijp KC, Zwarts MJ, Overeem S, van der Laak JA, et al. Intramuscular fibrous tissue determines muscle echo intensity in amyotrophic lateral sclerosis. Muscle Nerve. 2012 Mar;45(3):44950.

6 Akazawa N, Harada K, Okawa N, Tamura K, Moriyama $\mathrm{H}$. Muscle mass and intramuscular fat of the quadriceps are related to muscle strength in non-ambulatory chronic stroke survivors: a cross-sectional study. PLoS One. 2018 Aug 2;13(8):e0201789.

7 Maeda $\mathrm{H}$, Imada $\mathrm{K}$, Ishida $\mathrm{K}$, Akima $\mathrm{H}$. Quadriceps thickness and echo intensity predict gait independence in individuals with severe and mild hemiparetic stroke. Eur Neurol. 2020;83(2): 16773.

8 Akazawa N, Harada K, Okawa N, Kishi M, Tamura K, Moriyama H. Changes in quadriceps thickness and echo intensity in chronic stroke survivors: a 3-year longitudinal study. J Stroke Cerebrovasc Dis. 2021 Mar;30(3): 105543.

9 Kokura Y, Kato M, Taniguchi Y, Kimoto K, Okada Y. Energy intake during the acute phase and changes in femoral muscle thickness in older hemiplegic inpatients with stroke. Nutrition. 2020 Feb;70:110582.

10 Leszczak J, Czenczek-Lewandowska E, Przysada G, Wyszyńska J, Weres A, Baran J, et al. Diet after stroke and its impact on the components of body mass and functional fitness-A 4-month observation. Nutrients. 2019 May 29;11(6):1227.

11 Choe MA, An GJ, Lee YK, Im JH, Choi-Kwon $S$, Heitkemper M. Effect of inactivity and undernutrition after acute ischemic stroke in a rat hindlimb muscle model. Nurs Res. 2004 SepOct;53(5):283-92.
12 Springer J, Schust S, Peske K, Tschirner A, Rex A, Engel $\mathrm{O}$, et al. Catabolic signaling and muscle wasting after acute ischemic stroke in mice: indication for a stroke-specific sarcopenia. Stroke. 2014 Dec;45(12): 3675-83.

13 Lyden P, Brott T, Tilley B, Welch KM, Mascha EJ, Levine $S$, et al. Improved reliability of the NIH Stroke Scale using video training. NINDS TPA Stroke Study Group. Stroke. 1994 Nov;25(11):2220-6.

14 Charlson ME, Pompei P, Ales KL, MacKenzie CR. A new method of classifying prognostic comorbidity in longitudinal studies: development and validation. J Chronic Dis. 1987; 40(5):373-83.

15 Crary MA, Mann GD, Groher ME. Initial psychometric assessment of a functional oral intake scale for dysphagia in stroke patients. Arch Phys Med Rehabil. 2005 Aug;86(8): 1516-20.

16 Kaiser MJ, Bauer JM, Ramsch C, Uter W, Guigoz Y, Cederholm T, et al. Validation of the mini nutritional assessment short-form (MNA-SF): a practical tool for identification of nutritional status. J Nutr Health Aging. 2009 Nov; 13:782-8.

17 Brunnstrom S. Motor testing procedures in hemiplegia: based on sequential recovery stages. Phys Ther. 1966 Apr;46(4):357-75. 
18 Kawasaki Y, Sakai M, Nishimura K, Fujiwara K, Fujisaki K, Shimpo M, et al. Criterion validity of the visual estimation method for determining patients' meal intake in a community hospital. Clin Nutr. 2016 Dec;35(6): $1543-9$.

19 Roza AM, Shizgal HM. The Harris Benedict equation reevaluated: resting energy requirements and the body cell mass. Am J Clin Nutr. 1984 Jul;40(1):168-82.

20 Nagano A, Yamada Y, Miyake H, Domen K, Koyama T. Comparisons of predictive equations for resting energy expenditure in patients with cerebral infarct during acute care. J Stroke Cerebrovasc Dis. 2015 Aug;24(8): 1879-85.

21 Akima H, Yoshiko A, Tomita A, Ando R, Saito A, Ogawa M, et al. Relationship between quadriceps echo intensity and functional and morphological characteristics in older men and women. Arch Gerontol Geriatr. 2017 MayJun;70:105-11.
22 Nozoe M, Kanai M, Kubo H, Kitamura Y, Yamamoto M, Furuichi A, et al. Changes in quadriceps muscle thickness, disease severity, nutritional status, and C-reactive protein after acute stroke. J Stroke Cerebrovasc Dis. 2015 Aug;24:1879-85.

23 Taniguchi M, Yamada Y, Fukumoto Y, Sawano S, Minami S, Ikezoe T, et al. Increase in echo intensity and extracellular-to-intracellular water ratio is independently associated with muscle weakness in elderly women. Eur J Appl Physiol. 2017 Oct;117(10):2001-7.

24 Wilhelm EN, Rech A, Minozzo F, Radaelli R, Botton CE, Pinto RS. Relationship between quadriceps femoris echo intensity, muscle power, and functional capacity of older men. Age. 2014 Jun;36(3):9625.

25 Fairchild AJ, McDaniel HL. Best (but oft-forgotten) practices: mediation analysis. Am J Clin Nutr. 2017 Aug;106:708.

26 Fukumoto Y, Yamada Y, Ikezoe T, Watanabe $\mathrm{Y}$, Taniguchi M, Sawano S, et al. Association of physical activity with age-related changes in muscle echo intensity in older adults: a 4-year longitudinal study. J Appl Physiol. 2018 Nov 1;125(5):1468-74.
27 Yeh DD, Ortiz-Reyes LA, Quraishi SA, Chokengarmwong N, Avery L, Kaafarani HMA, et al. Early nutritional inadequacy is associated with psoas muscle deterioration and worse clinical outcomes in critically ill surgical patients. J Crit Care. 2018 Jun;45:7-13.

28 Deutz NE, Bauer JM, Barazzoni R, Biolo G, Boirie Y, Bosy-Westphal A, et al. Protein intake and exercise for optimal muscle function with aging: recommendations from the ESPEN Expert Group. Clin Nutr. 2014 Dec; 33(6):929-36.

29 Hafer-Macko CE, Ryan AS, Ivey FM, Macko RF. Skeletal muscle changes after hemiparetic stroke and potential beneficial effects of exercise intervention strategies. J Rehabil Res Dev. 2008;45(2):261-72.

30 Springer J, Schust S, Peske K, Tschirner A, Rex A, Engel O, et al. Catabolic signaling and muscle wasting after acute ischemic stroke in mice: indication for a stroke-specific sarcopenia. Stroke. 2014 Dec;45(12):3675-83. 